\title{
GPPS-BJ-2019-24
}

\section{Nonlinear Dynamics of a Rod-Fastened Rotor}

\author{
Qi Da* \\ Institute of Turbomachinery, Xi'an Jiaotong \\ University; \\ Shaanxi Engineering Laboratory of \\ Turbomachinery and Power Equipment \\ daqi_1995@163.com \\ Xi'an, Shaanxi, P. R. China \\ Pu Li \\ Institute of Turbomachinery, Xi'an Jiaotong \\ University; \\ Shaanxi Engineering Laboratory of \\ Turbomachinery and Power Equipment \\ lipu1215@xjtu.edu.cn \\ Xi'an, Shaanxi, P. R. China
}

\begin{abstract}
Nonlinear bending stiffness of a rod-fastened rotor varies with applied loads posing a significant influence on the dynamic behavior. In this paper, the cubic model is adopted to perform an analysis of the nonlinear dynamic characteristics of a rod-fastend rotor: Firstly, the bending stiffness of a rod-fastened rotor involving two separate disks is obtained making use of the 3D FEM model. Then, a cubic stiffness model is applied to approximate the nonlinear stiffness and subsequently the corresponding governing equation can be derived. The harmonic balance method(HBM) as well as an iterative optimization method based on Broyden algorithm are adopted to yield the steady state response. Finally, influences of different parameters on rotor dynamics are further investigated in this manuscript. The results show that the cubic model can be used to demonstrate the amplitude jump behavior of the rod-fastened rotor and has an abundant nonlinear behavior. Furthermore, by adjusting the nonlinear system parameters, the position of the unstable region in the amplitude-frequency response curve can be changed, thereby effectively preventing the rotor system from operating in an unstable frequency region.
\end{abstract}

\section{INTRODUCTION}

Rod-fastened rotors are widely used in heavy-duty gas turbines and aero-engines to ensure relative static between disks and a certain stiffness of the disk segments by applying a preload on the bolts. However, the joint structure complicates the dynamic behaviors of rotor because of its internal contact friction nonlinearities, e.g., the change of stability, the jump phenomenon.[1] Yuan et al. [2] showed that, even with a small bending moment,

\author{
Qi Yuan \\ Institute of Turbomachinery, Xi'an Jiaotong \\ University; \\ Shaanxi Engineering Laboratory of \\ Turbomachinery and Power Equipment \\ qyuan@xjtu.edu.cn \\ Xi'an, Shaanxi, P. R. China \\ Yang Liu \\ Institute of Turbomachinery, Xi'an Jiaotong \\ University; \\ Shaanxi Engineering Laboratory of \\ Turbomachinery and Power Equipment \\ ly.2008@stu.xjtu.edu.cn \\ Xi'an, Shaanxi, P. R. China
}

the rotor disk contact surface will still be partially separated, resulting in a decrease of the overall stiffness of the rotor. Qin, Z. Y et al. [3] studied the stiffness of the drum rotor contact surface of an aero-engine. The results show that the relative rotation angle between the disks exists a nonlinear variation region with the linear increase of the bending moment. Liu, Z. Q et al. [4] used a piecewise linear model of angular stiffness to approximate the stiffness variation of the bolts in the establishment of the model of aero-engine rotor. Compared with the continuous rotor(ie, the rotor without rod fastened rotor), the dynamic response of the piecewise linear stiffness model is quite different from that of the linear stiffness model. Cheng et al. [5, 6] considered the effects of the disk contact surfaces and the bolts on the stiffness of rotor and established a rotor model with cubic stiffness to explain the amplitude "jump" phenomenon in the dynamic response of the rod-fastened rotor. Mat Isa, A. A et al. [7] showed that the stiffness of a rod-fastened rotor changes nonlinearly as bending moment increases, and the response of the rotor is complicated.

Under certain conditions, the amplitude "jump" phenomenon which is not present in linear systems in the amplitude-frequency response causes frequent rotor failures and huge economic losses. Cveticanin, $\mathrm{L}$ et al. [810] studied the amplitude "jump" phenomenon of nonlinear rotor systems, regarding it as a kind of bad working condition of the rotor, and try to avoid it. In industry, increasing the preloading can inhibit the "jump" phenomenon because it makes contact surface harder to seperate. Commonly, the nonlinearities of such rotors are neglected or linearized in the industrial applications for 
the large scale system[11] and there are few researches on the nonlinear vibration characteristics of rod-fastened rotors. Most of the previous researches use the stiffness hardening model, that is, the overall stiffness of bolt increases with the increase of the bending moment. However, there is not a clear conclusion about the "jump" phenomenon in the amplitude-frequency response.

In this paper, firstly, the FEM is used to calculate a nonlinear stiffness curve of the bolt and the contact surface that indicates the variation law of the equivalent stiffness as bending moment increases; Then the cubic stiffness model is used to fit the nonlinear stiffness curve. Finally, through numerical simulation, the influences of different parameters of the model on the vibration characteristics of the rotor is analyzed to avoid the "jump" phenomenon.

\section{METHODOLOGY}

Figure 1 is a rod-fastened rotor model consisting of a shaft head, a hub, a disk, a tie rod and a frictional contact surface, where $2 l_{1}$ represents the length of segment of disk, $l_{2}$ represents the length of the shaft, $l_{3}$ and $l_{4}$ respectively represent the axial length of the hub and the disk, and $2 l$ represents the totel length of the model. The bending stiffness of the shaft head, the hub and the disk are expressed respectively as $K_{s}, K_{h}$ and $K_{d s} . K_{r}$ represents a stiffness in parallel with rods and contact surface.

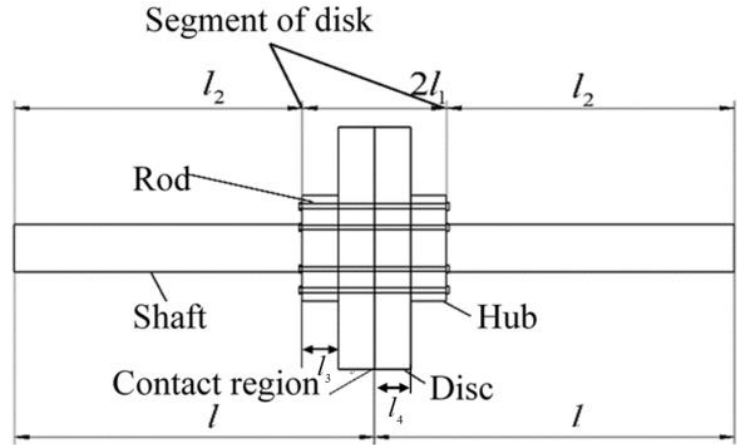

(a) Structure of a rod-fastened rotor

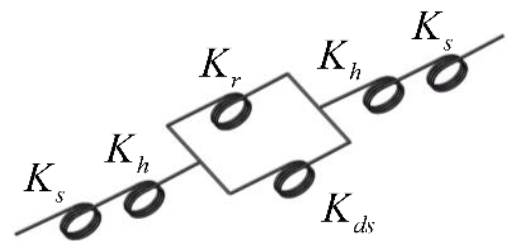

\section{(b) Stiffness model of a rod-fastened rotor}

Figure 1 Model of a rod-fastened rotor

The stiffness of the rotor disk segment that contains the effect of the frictional contact surface can be expressed as

$$
K_{c}=\left(\frac{1}{K_{h}}+\frac{1}{K_{d s}+K_{r}}\right)^{-1}
$$

The bending stiffness of the disk segment is calculated by FEM, according to the relationship between bending moment and rotation angle

$$
K_{f i j}=\frac{M_{j}}{\varphi_{i}}
$$

where $K_{f i j}$ is the bending moment applied in the $j$ direction when the disk has a unit rotation angle in the $i$ direction.

To simplify the analysis, the disk is considered to be isotropic, not counting the stiffness in the cross direction.

Then the bending stiffness can be expressed as

$$
K_{f i j}=\delta_{i j} \frac{M_{j}}{\varphi_{i}}
$$

Where $\delta_{i j}$ is the Kroneck function, which means

$$
\delta_{i j}= \begin{cases}1, & i=j \\ 0, & i \neq j\end{cases}
$$

From Eq. (3), the bending stiffness of the disk segment $K_{c}$, which contains the effect of the frictional contact surface, can be calculated by FEM.

The lateral stiffness of the disk segment $K_{c l}$ is derived by the moment-area method

$$
K_{c l}=\left[\frac{l_{3}\left(2 l_{3}+3 l_{4}\right)}{12 K_{h}}+\frac{3 l_{3}^{2}-3 l_{3} l_{4}+4 l_{4}^{2}}{48\left(K_{d s}+K_{r}\right)}\right]^{-1}
$$

The lateral stiffness of the $\operatorname{hub}\left(K_{h l}\right)$ and the $\operatorname{disk}\left(K_{d s l}\right)$ are also obtained by the moment-area method. Then the lateral stiffness in parallel with the tie rod and the contact surface $K_{r l}$ can be obtained based on the relationship between $K_{c l}, K_{h l}, K_{d s l}$ and $K_{r l}$ (see Eq. (1))

$$
K_{r l}=\left(\frac{1}{K_{c l}}-\frac{1}{K_{h l}}\right)^{-1}-K_{d s l}
$$

Since the relative rotation angle of the two disks is small, the relative lateral displacement between two disks $X$ is approximately expressed as $\varphi l$, where $l$ is the distance from the contact surface to the shaft head (see Figure 2).

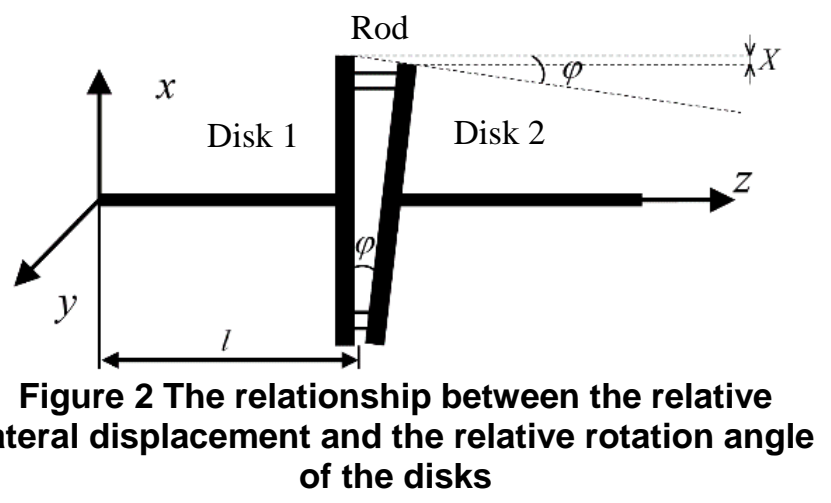

The lateral restoring force of $K_{r l}$ is expressed as:

$$
F_{r l}=K_{r l} \cdot X
$$

The corresponding 3-D model is established by the finite element software ANSYS (Figure 3). The parameters of this model are shown in Table 1. 

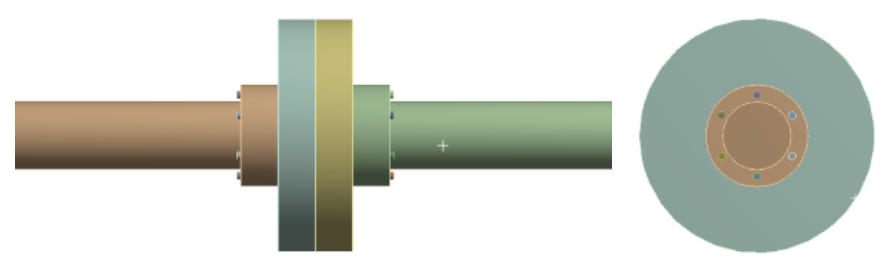

Figure 3 3-D finite element model of the rodfastened rotor

According to the method used in reference [12], the relationship between the relative rotation angle of the disks and the bending moment is calculated using Eq. (3) (see Figure 4). Then, from Eq. (5) and (6), the relationship between $K_{r l}$ and $X$ is obtained (see Figure 5).

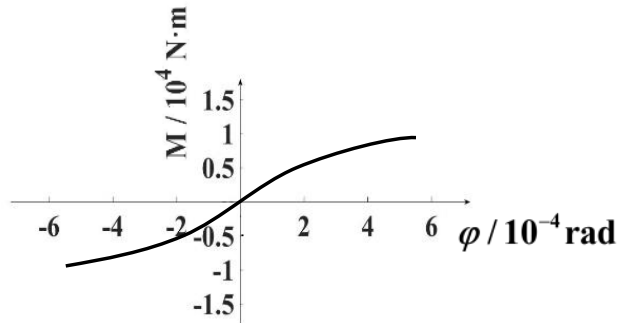

Figure 4 The relationship between the relative rotation angle of the disks and the bending moment

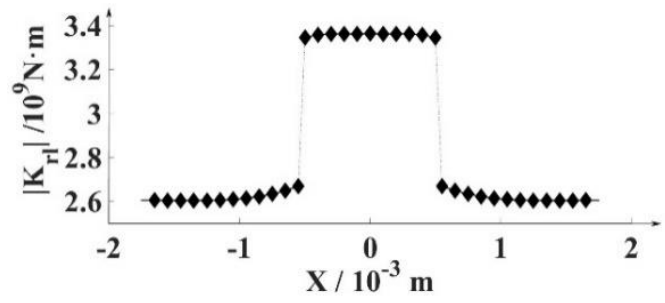

Figure 5 the relationship between the lateral stiffness $K_{r l}$ and the relative lateral displacement $X$

TABLE 1 PYSICAL PARAMETERS OF THE RODFASTENED ROTOR

\begin{tabular}{|c|c|c|c|c|}
\hline $\begin{array}{l}\text { Para. } \\
\text { No. }\end{array}$ & Name & Symbol & Unit & Value \\
\hline 1 & Number of tie rods & $N$ & 1 & 6 \\
\hline 2 & Diameter of tie rods & $d_{r}$ & $\mathrm{~m}$ & 0.012 \\
\hline 3 & $\begin{array}{l}\text { Young's modulus of } \\
\text { elasticity }\end{array}$ & $E$ & $\mathrm{GN} \cdot \mathrm{m}^{-2}$ & 206 \\
\hline 4 & Material density & $\rho$ & $\mathrm{kg} \cdot \mathrm{m}^{3}$ & 7870 \\
\hline 5 & Rotor mass & $m$ & $\mathrm{~kg}$ & 885.28 \\
\hline 6 & Damping ratio & $\zeta$ & & 0.02 \\
\hline 7 & Pitch radius & $r_{p}$ & $\mathrm{~m}$ & 0.1075 \\
\hline 8 & Shaft length & $l_{2}$ & $\mathrm{~m}$ & 0.8 \\
\hline 9 & Disk segment length & $l_{1}$ & $\mathrm{~m}$ & 0.2 \\
\hline 10 & Total pretightening force & $F_{\text {pre }}$ & $N$ & 120000 \\
\hline 11 & $\begin{array}{l}\text { Frictional Coefficient of } \\
\text { the contact surface }\end{array}$ & / & / & 0.1 \\
\hline
\end{tabular}

\section{DYNAMIC EQUATIONS OF ROTOR}

The disks are simplified to two rigid disks with mass $m_{1}$ and $m_{2}$ respectively, and the eccentricity of the two disks is expressed as $e$.the angle of the two eccentricity vectors of disks is expressed as $\theta$. The rod and the contact surface are equivalent to a bending spring. Both ends of the disk segment are respectively connected with an elastic shaft, and the lateral stiffness of the two disks is expressed as $k_{1}$ and $k_{2}$ respectively. The damping coefficients of the left and right end shafts and the bending spring are expressed as $c_{1}, c_{2}$ and $c_{3}$. The simplified model is shown in Figure 6 . The displacement vector of a single disk in this model is expressed as

$$
q_{i}=\left[\begin{array}{ll}
x_{i} & y_{i}
\end{array}\right]^{\mathrm{T}}
$$

where, $x$ and $y$ are the two degrees of freedom of a single disk(see Figure 2).

The equation of motion of the model above is established according to the D'Alembert principle:

$$
\begin{aligned}
& m_{1} \ddot{x}_{1}+c_{1} \dot{x}_{1}+c_{3}\left(\dot{x}_{1}-\dot{x}_{2}\right)+k_{1} x_{1}+F\left(x_{1}-x_{2}\right)=m_{1} e \Omega^{2} \cos (\Omega t) \\
& m_{2} \ddot{x}_{2}+c_{2} \dot{x}_{2}-c_{3}\left(\dot{x}_{1}-\dot{x}_{2}\right)+k_{2} x_{2}-F\left(x_{1}-x_{2}\right)=m_{2} e \Omega^{2} \cos (\Omega t+\theta) \\
& m_{1} \ddot{y}_{1}+c_{1} \dot{y}_{1}+c_{3}\left(\dot{y}_{1}-\dot{y}_{2}\right)+k_{1} y_{1}+F\left(y_{1}-y_{2}\right)=m_{1} e \Omega^{2} \sin (\Omega t) \\
& m_{2} \ddot{y}_{2}+c_{2} \dot{y}_{2}-c_{3}\left(\dot{y}_{1}-\dot{y}_{2}\right)+k_{2} y_{2}-F\left(y_{1}-y_{2}\right)=m_{2} e \Omega^{2} \sin (\Omega t+\theta)
\end{aligned}
$$

Where, $\Omega$ is the rotating speed of the rotor and $F(\cdot)$ represents the function of the nonlinear restoring force of the bending spring.

The matrix form of Eq. 9 is expressed as

$$
M X^{\prime \prime}+C X^{\prime}+K X=f(t, X)
$$

where,

$$
\begin{aligned}
& X=\left(\begin{array}{l}
x_{1} \\
x_{2} \\
y_{1} \\
y_{2}
\end{array}\right) \quad, \quad C=\left(\begin{array}{cccc}
c_{1}+c_{3} & -c_{3} & & \\
-c_{3} & c_{1}+c_{3} & & \\
& & c_{1}+c_{3} & -c_{3} \\
& & -c_{3} & c_{1}+c_{3}
\end{array}\right) \\
& K=\left(\begin{array}{llll}
k_{1} & & & \\
& k_{2} & & \\
& & k_{1} & \\
& & & k_{2}
\end{array}\right) \quad, \quad M=\left(\begin{array}{llll}
m_{1} & & & \\
& m_{2} & & \\
& & m_{1} & \\
& & & m_{2}
\end{array}\right) \\
& f(t, X)=e \Omega^{2}\left(\begin{array}{c}
m_{1} \cos (\Omega t) \\
m_{2} \cos (\Omega t+\theta) \\
m_{1} \sin (\Omega t) \\
m_{2} \sin (\Omega t+\theta)
\end{array}\right)-\left(\begin{array}{c}
F\left(x_{1}-x_{2}\right) \\
F\left(x_{2}-x_{1}\right) \\
F\left(y_{1}-y_{2}\right) \\
F\left(y_{2}-y_{1}\right)
\end{array}\right)
\end{aligned}
$$

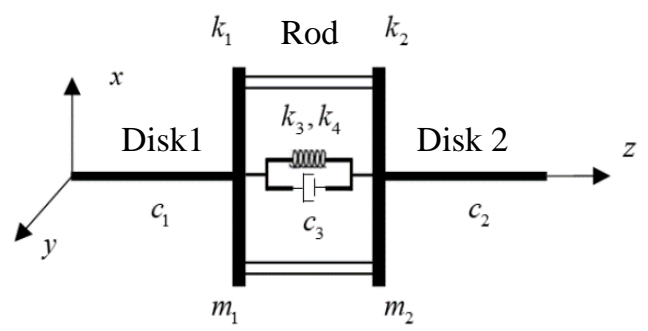

Figure 6 The simplified model of the rod-fastened rotor

\section{CUBIC NONLINEAR STIFFNESS MODEL}

As can be seen from Figure 4 , as the moment load increases, the stiffness of the bending spring changes nonlinearly. As it shows in Figure 4, the relatonship is very 
much like a cubic function, so that, the function of the restoring force in direction $x$ can be expressed as

$$
F(x)=k_{3} x-k_{4} x^{3}
$$

where, $k_{3}$ is the linear stiffness of the bending spring, and $k_{4}$ is the nonlinear stiffness of the bending spring representing the weakening effect of the reduction of the contact area of the disks to the lateral stiffness of the two disks.

\section{Dynamics equation of simplified model}

The Eq. (9) is uncoupled in the direction and duing to its symmetry, so that only the lateral vibration in the $x$ direction is studied.

Define $\tau=\Omega t, \quad x_{3}=x_{2}-x_{1}, r_{j}=x_{j} / e(j=1,2,3)$ (which means dimensionless amplitude) and $\beta_{2}, \beta_{3}, \beta_{4}$ (which can be calculated from $k_{1}=\beta_{2} k_{2}=\beta_{3} k_{3}=\beta_{4} k_{4}$ ) and rewrite Eq. (9) as a dimensionless form as follows

$$
\begin{array}{r}
r_{2}^{\prime \prime}+r_{3}^{\prime \prime}+\frac{2 \zeta_{1}}{\lambda}\left(r_{2}^{\prime}+r_{3}^{\prime}\right)+\frac{2 \zeta_{3}}{\lambda} r_{3}^{\prime}+\frac{1}{\lambda^{2}}\left(r_{2}+r_{3}\right)+\frac{\beta_{3}}{\lambda^{2}} r_{3}-\frac{\xi}{\lambda^{2}} r_{3}^{3} \\
=\cos (\tau) \\
r_{2}^{\prime \prime}+\frac{2 \zeta_{2}}{\alpha \lambda} r_{2}^{\prime}-\frac{2 \zeta_{3}}{\alpha \lambda} r_{3}^{\prime}+\frac{\beta_{2}}{\alpha \lambda^{2}} r_{2}-\frac{\beta_{3}}{\alpha \lambda^{2}} r_{3}+\frac{\xi}{\alpha \lambda^{2}} r_{3}^{3}=\cos (\tau+\theta)
\end{array}
$$

where,

$$
r^{\prime}=\frac{d r}{d \tau}, \quad r^{\prime \prime}=\frac{d^{2} r}{d \tau^{2}}(i=1,2,3), \text { which mean the first }
$$

and second derivative of $r$ to $\tau$;

$\alpha=m_{2} / m_{1}$, which means the disk mass ratio;

$\omega_{1}=\sqrt{k_{1} / m_{1}}$, which means the natural frequency of the disk 1 ;

$$
\zeta_{j}=c_{j} /\left(2 m_{1} \omega_{1}\right)(j=1,2,3) \text {, which mean damping }
$$

ratios;

$\Omega / \omega_{1}=\lambda$, which means rotating speed ratio;

$\xi=e^{2} \beta_{4}$, which means the ratio of nonlinear stiffness to linear stiffness, defined as stiffness ratio.

The harmonic balance method(HBM) is discussed in detail in reference [13] and it is suit well for the computation of periodic solutions of ODEs. In this paper, HBM is used to solve the above differential equations approximately, and the state response of the equations can be expressed in the complex Fourious series as

$$
r_{j}=\sum_{n=-\infty}^{+\infty} \hat{r}_{j n} e^{i n \Omega t} \text { ( } n \text { is an integer) }
$$

where $\hat{r}_{j n}$ are the amplitudes of the harmonics of $r_{j}$. Since the amplitude of the high order harmonics is extremely small, the series is truncated after $n= \pm 3$.

Substituting Eq. (13) into Eq. (12) leads to the algebraic equations corresponding to the differential equations

$$
\boldsymbol{f}(\boldsymbol{r})=\left\{\begin{array}{c}
f_{1}\left(r_{11}, r_{12}, \cdots, r_{16}, r_{21}, r_{22}, \cdots, r_{26}\right)=0 \\
f_{2}\left(r_{11}, r_{12}, \cdots, r_{16}, r_{21}, r_{22}, \cdots, r_{26}\right)=0 \\
\vdots \\
f_{13}\left(r_{11}, r_{12}, \cdots, r_{16}, r_{21}, r_{22}, \cdots, r_{26}\right)=0
\end{array}\right.
$$

Then, an iterative algorithm is used to solve the above algebraic equation to obtain an approximate solution of the differential equations.

\section{Optimization algorithm for nonlinear algebraic equations}

\section{(a) Broyden algorithm}

The Broyden algorithm has a superlinear convergence rate and converges fast under certain conditions $[14,15]$. The method iterative process is expressed as

$$
\begin{gathered}
\tilde{r}^{(k+1)}=\tilde{r}^{(k)}-A_{k}^{-1} f\left(\tilde{r}^{(k)}\right) \\
A_{k} \tilde{s}_{k}=\tilde{y}_{k}
\end{gathered}
$$

Where $\tilde{r}^{(k)}$ represents the value of the certain column vector to be solved in step $\mathrm{k}, f\left(\tilde{r}^{(k)}\right)$ represents the residual of equtions in the step $\mathrm{k}, A_{k}$ is the Jacobian matrix of the equtions in step $\mathrm{k}$ and $s_{k}=\tilde{r}^{(k)}-\tilde{r}^{(k-1)}, \quad y_{k}=f\left(\tilde{r}^{(k)}\right)-f\left(\tilde{r}^{(k-1)}\right)$.

Give $A_{0}=J_{f}\left(\tilde{r}^{(0)}\right)$ and update $A_{k}$ during the iteration process with the following formular

$$
A_{k}^{-1}=A_{k-1}^{-1}+\frac{\left(\tilde{s}^{(k)}-A_{k-1}^{-1} \tilde{y}^{(k)}\right) \tilde{s}^{(k) T} A_{k-1}^{-1}}{\tilde{s}^{(k) T} A_{k-1}^{-1} \tilde{y}^{(k)}}
$$

The solution is considered to converge when the residual is less than the residual limit(i.e., eps )

$$
\left|f\left(\tilde{r}^{k}\right)\right| \leq e p s \text { or }\left|\tilde{s}_{k}\right| \leq e p s
$$

\section{(b) Least Squares method}

Eq. 14 has 13 algebraic equations but only 12 amplitude coefficients. In order to solve the hyperstatic problem of equations, the least squares method [14] is used, and the the residual limit is set to be sufficiently small (e.g., $1.0 \times 10^{-10}$ ) to ensure the accuracy of the solution. The iterative process after using the least squares method is expressed as

$$
\tilde{r}^{(k+1)}=\tilde{r}^{(k)}-\left(A_{k}^{T} A_{k}\right)^{-1}\left(A_{k}^{T} f\left(\tilde{r}^{(k)}\right)\right)
$$

\section{(c) Method for reducing the condition number of the coefficient matrix}

For Eq. 9, the order of the corresponding algebraic nonlinear equations(ie, Eq. 14) to be solved is large, and the higher-order harmonic amplitude is too small compared to that of first-order; therefore, when the value of $\Omega$ is large, the condition number of the coefficient matrix is too high(e.g., $10^{9}$ ), making the matrix inversion extremely timeconsuming and often fail. Numerical simulations show that the condition number is related to the value of some parameters in the equations. By using the dimensionless amplitude-frequency response curve $\lambda-r$ instead of the amplitude-frequency response curve $\Omega-x$, the range of $\Omega$ can be scaled $\operatorname{down}\left(\lambda=\Omega / \omega_{1}\right)$, and then the equations can be solved. That is the meaning of nondimensionalization of Eq. 9(see Eq. 12).

Then, a continuous algorithm is used to search unstable solution[16] of Eq. 14.

\section{(d) Procedure for solving the equations}

The procedure for calculating $r$ is shown in Figure 7, where $r_{0}$ is the initial value of $r, s$ is the step value of rotating speed, $\Omega_{0}$ and $\Omega_{\text {end }}$ mean the start and end value of rotaing speed respectively. 


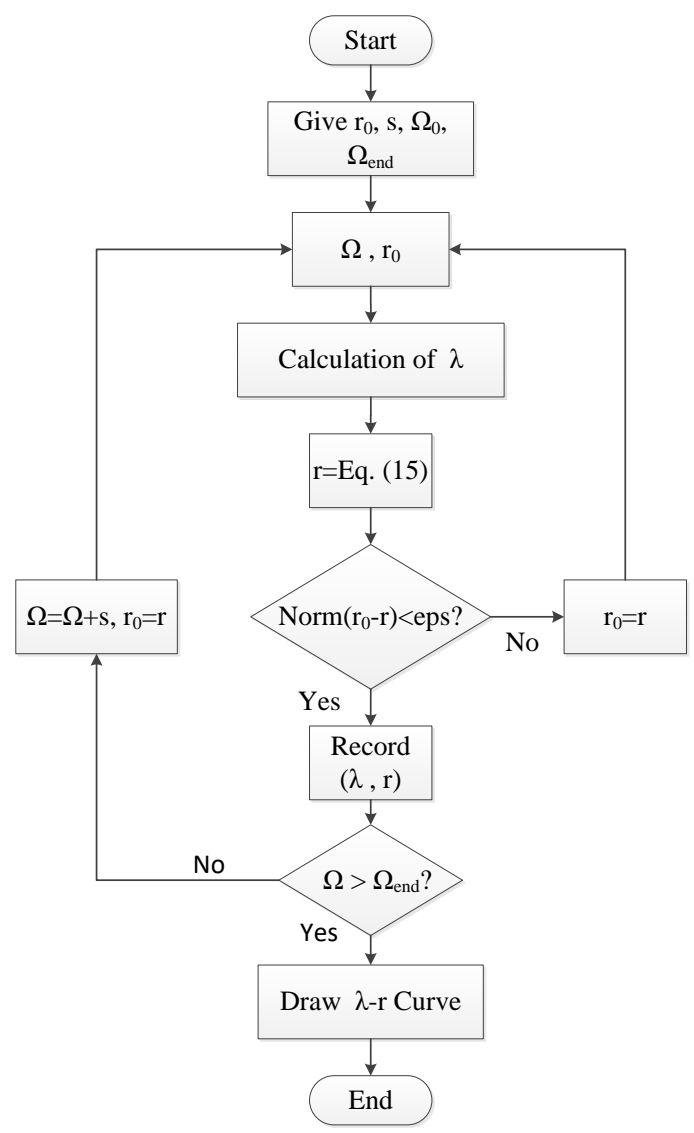

Figure 7 Calculation flow chart of amplitude frequency curve of model rotor

\section{Calculation results and analysis}

The parameters used in the calculation are shown in Table 2. According to the calculation results, the dimensionless amplitude-frequency response curve of the rod-fastened rotor during the acceleration and deceleration is drawn. Then change the parameters to analyze the effects of different parameters of the model on the response curves.

\section{(a) The amplitude-frequency response curves of disks}

Take the first group of parameters in Table 2 (case 1), and draw the dimensionless amplitude-frequency response curve during the acceleration and deceleration (see Figure 8).

TABLE 2 DIFFERENT CASES FOR PARAMETERS ANALYSIS

\begin{tabular}{cccccccc}
\hline case & $\alpha$ & $\beta_{2}$ & $\beta_{3}$ & $\begin{array}{c}\beta_{4} \\
/ 10^{5} \mathrm{~m}^{-2}\end{array}$ & $\theta /{ }^{\circ}$ & $e / \mu \mathrm{m}$ & $\begin{array}{c}\zeta_{i} \\
/ 10^{-3}\end{array}$ \\
\hline 1 & 0.75 & 1 & 1 & 5 & 90 & 10 & 5.7 \\
2 & 0.75 & 1 & 1 & 0 & 90 & 10 & 5.7 \\
3 & 0.75 & 1 & 1 & 5 & 90 & 11 & 5.7 \\
4 & 0.75 & 1 & 1 & 5 & 90 & 12 & 5.7 \\
5 & 0.75 & 1 & 1 & 5 & 90 & 13 & 5.7 \\
6 & 0.75 & 1 & 1 & 5 & 60 & 10 & 5.7 \\
7 & 0.75 & 1 & 1 & 5 & 70 & 10 & 5.7 \\
8 & 0.75 & 1 & 1 & 5 & 80 & 10 & 5.7 \\
9 & 0.75 & 1 & 1 & 5 & 90 & 10 & 6.0
\end{tabular}

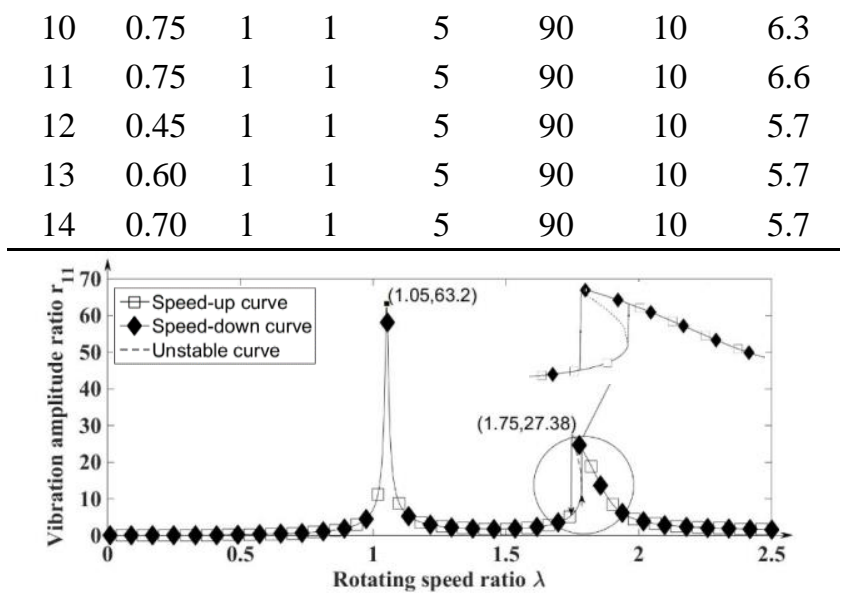

(a) First-order harmonic curve of the disk 1

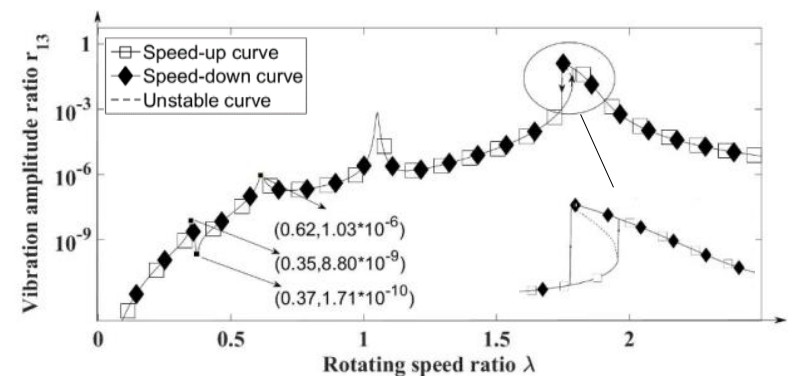

(b) Third-order harmonic curve of the disk 1

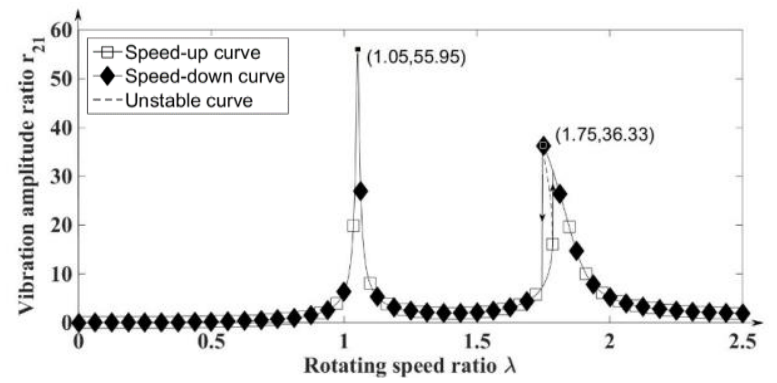

(c) First-order harmonic curve of the disk 2

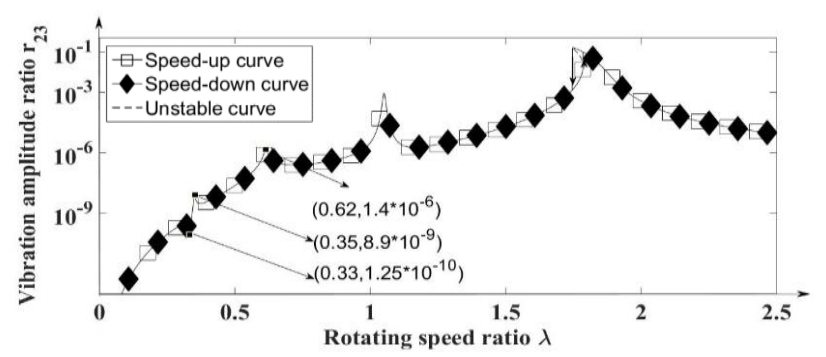

(d) Third-order harmonic curve of the disk 2

\section{Figure 8 The dimensionless amplitude-frequency response curve}

The Figure above shows the first-order and third-order harmonics of the dimensionless amplitude-frequency curve, and the second-order harmonic amplitude is too small (not shown in the logarithmic graph). The solutions of Eq. (12) at each rotaing speed constitude the dimension-frequency response curves of two-disks whose nonliner characters are in good agreement with that in perivous work. Both simulation and experimental results show that the second- 
formant of the curves tilt to the left or right while the other part of the curves hardly change comparing with the curves when the nonlinear stiffness is not included[2,5,7].

It can be seen that the dimension-frequency response curves of two-disks have the same trend with the dimensionless rotaing speed increases, but the amplitudes are different.

\section{(b) Effect of nonlinear stiffness on vibration \\ characteristics}

In Table 2, comparing case 2, which means no nonlinear stiffness term in restoring force function, with case 1 , the nonlinear stiffness causes a significant change in the secondorder natural frequency of disk 1 , but the first-order natural frequency is not changed much(see Figure 9).

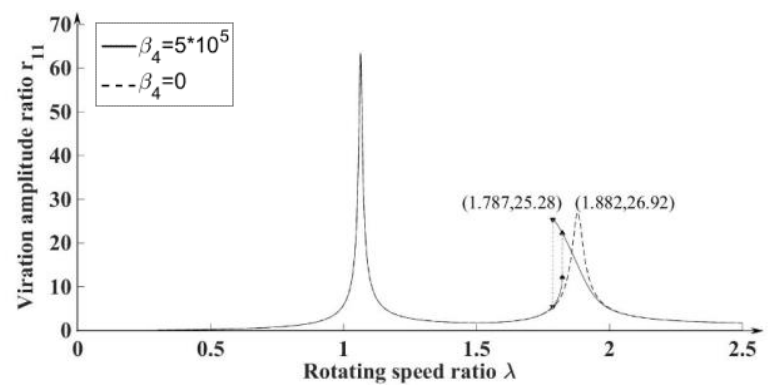

Figure 9 The effect of nonlinear stiffness on natural frequencies of disk 1

As can be seen from Figure 8, when $\lambda<1$, there are two peaks in each curve whose corresponding value of the abscissa are $1 / 3$ of the first- and second-order natural frequency of the disks.

The subharmonics indicates that the vibration response of cubic stiffness model is affected by the nonlinear stiffness term and exhibits different vibration response characteristics from the linear model.

In the first-order and third-order harmonic curves, the amplitude "jump" phenomenon can be observed, but the positions of "jump" in the speed-up and speed-down curves are different. The reason lies in the nonlinear term in the dynamic equation, which endures multiple solutions to the Eq. 12 at the same rotating speed ratio.

According to the stability judgment of the solution, the whole region of $\lambda$ can be divided into a stable solution region and an unstable solution region.

The number of solutions in unstable region is different from that of stable region, so as $\lambda$ increases or decreases, the number of the solution of Eq. 12 corresponging to a certain $\lambda$ changes.

Since the Eq. 12 only has multiple solutions in a specific range of $\lambda$ (which means the unstable region), when $\lambda$ increases or decreases, the corresponding rotating speed ratio of the amplitude "jump" position is different due to the position where $\lambda$ enters the unstable region.

\section{(c) Effect of parameters on vibration characteristics}

In order to analyze the amplitude "jump" phenomenon of the response, it is necessary to study the effect of parameters on the response curve.
Take the parameters in Table 2 and compare the dimensionless amplitude-frequency response curves with different values of the eccentricity, the eccentricity angle and the damping ratio (see Figure 10).

Given that the responses of the left and right disks are similar, the nonlinear phenomenon mainly occurs at the second-order natural frequency, and the amplitude of secondorder and third-order harmonics are too small compared to that of the first-order harmonic, so only the first-order of dimensionless amplitude-frequency response curve of the disk 1 near the second-order natural frequency is drawn.

As can be seen from Figure 10, increasing the eccentricity, increasing the angle of the eccentricities between the two disks, or reducing the damping ratio will cause the second-formant to tilt to the left and expand the region of the multi-solution.

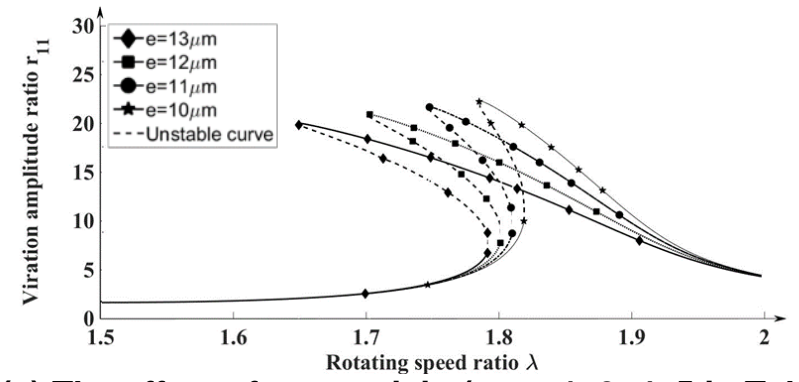

(a) The effect of eccentricity(case 1, 3, 4, 5 in Table 2)

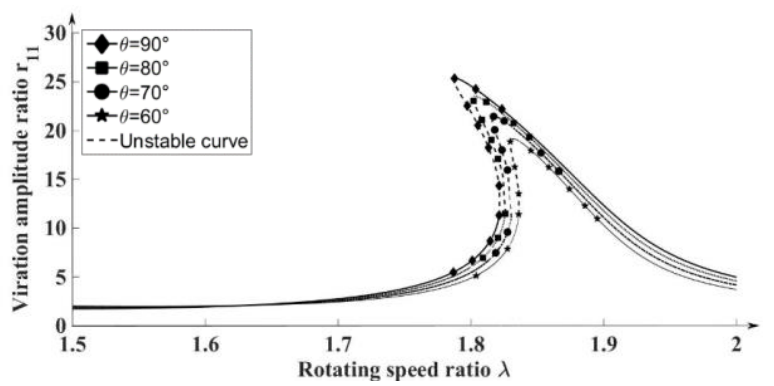

(b) The effect of the angle of the eccentricities(case 1, 6, 7, 8 in Table 2)

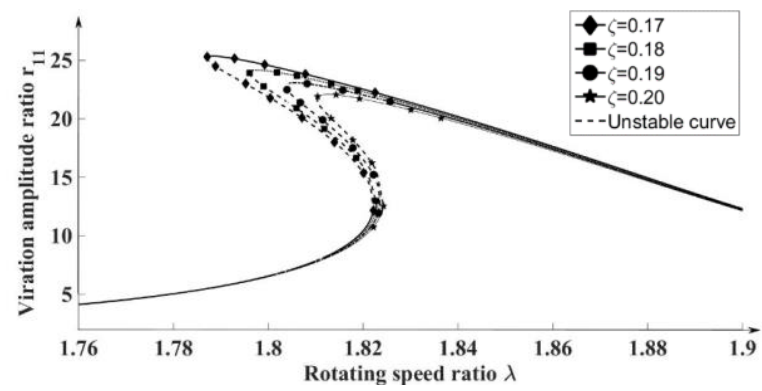

(c) The effect of damping ratio(case 1, 9, 10, 11 in Table 2)

\section{Figure 10 Comparison of the effect of different parameters on the response curve}

As can be seen from Figure 11, increasing the mass ratio causes the reduction of first- and second-orders of the natural frequency of the of the disk 1 , and the value of amplitude corresponding to the second-order natural frequency increases. 
The non-dimensional amplitude-frequency curve of the disk 2 is not shown due to the limitation of the space, but the trend of the dimensionless amplitude of the resonance is opposite to that of the disk 1 , and the first-order formant value decreases with the increase of the mass ratio.

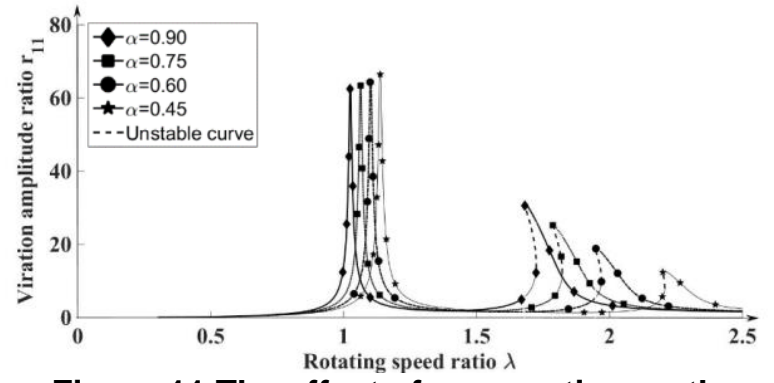

Figure 11 The effect of mass ratios on the dimensionless amplitude-frequency response curve(case 1, 12, 13, 14 in Table 2)

\section{(d) Effect of nonlinear stiffness ratio on vibration}

\section{characteristics}

In order to analyze the effect of nonlinear stiffness ratio(i.e., $\xi$ ) on response curve, take the parameters in Table 3 , and draw the response curves of disk 1 in different cases.

As can be seen from Figure 12, the second-order formant is tilted to the left as the nonlinear stiffness ratio increases. However, the first-order formant doesn't change because the nonlinear term(ie, $\left.k_{4} x_{3}{ }^{3}\right)$ is extremely small when the displacement difference(ie, $x_{3}$ ) between the two disks is small(point A in Figure 12), and the nonlinear stiffness term hardly affects the overall stiffness; As $x_{3}$ increases, the nonlinear stiffness increases rapidly and takes a maximum near the second-order natural frequency(point B in Figure 12). At this time, the stiffness has a strong nonlinearity.

The dotted line in Figure 12 shows the trend of first-order harmonic of the dimensionless displacement difference of the two disks(ie, $\left.r_{3}\right)$ as the rotating speed ratio increases.

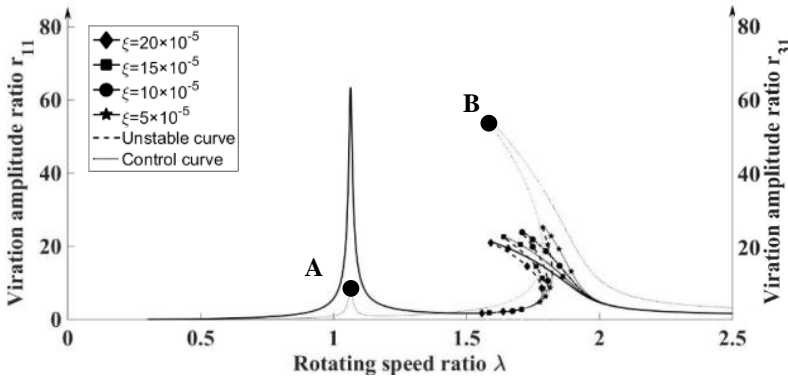

Figure 12 The effect of nonlinear stiffness ratio on the dimensionless amplitude-frequency response curve

TABLE 3 DIFFERENT CASES FOR NONLINEAR STIFFNESS RATIOS ANALYSIS

\begin{tabular}{ccccccc}
\hline case & $\alpha$ & $\beta_{2}$ & $\beta_{3}$ & $\theta /{ }^{\circ}$ & $e / \mu \mathrm{m}$ & $\xi / \times 10^{-5}$ \\
\hline 1 & 0.75 & 1 & 1 & 90 & 10 & 5 \\
2 & 0.75 & 1 & 1 & 90 & 10 & 10 \\
3 & 0.75 & 1 & 1 & 90 & 10 & 15 \\
4 & 0.75 & 1 & 1 & 90 & 10 & 20 \\
\hline
\end{tabular}

\section{RESULTS AND DISCUSSION}

Reference [1] uses classical and state of the art reduced order modeling (ROM) techniques to analyze jointed structures with local contact nonlinearities and the forced frequency responses of jointed structures are very similar to the responses of the simplified rotor model.

In this paper, the effects of parameters of the model on the dimensionless amplitude-frequency response curve can be analyzed from the perspective of the effects of energy and nonlinear stiffness:

(1) From Eq.s (9) and (12), increasing the value of eccentricity is equivalent to increasing the proportion of nonlinear stiffness and reducing the overall stiffness of the bending spring. Therefore, the unstable region of the response curve of the disk 1 expands, and the second-order natural frequency decreases.

(2) Increasing the eccentricity angle will increase the displacement difference (ie, $x_{3}$ or $r_{3}$ ) of the two disks, which can increase the nonlinear stiffness and then expands the unstable region of the response curve of the disk 1 ;

(3) When the external input energy is constant, as damping ratio increases, the energy consumed by the system increases in one cycle, and thus the amplitude of response decreases;

(4) It is known from the Eq. (12) that increasing the mass ratio is equivalent to increase the proportion of the mass of the disk 1. Therefore, the amplitude of the response curve increases and the natural frequencies of the disk decrease.

\section{CONCLUSION}

In this paper, the cubic stiffness model is used to approximate the equivalent lateral stiffness of the rod and the contact surface, and the rotor simplified model is established. From the calculation results of the simplified models, the following conclusions are obtained:

(1) The cubic stiffness models can simulate the nonlinear dynamics of the rod-fastened rotor and reflect the amplitude "jump" phenomenon during operation;

(2) The reason for the amplitude "jump" phenomenon of the response is that the dynamic equation has multiple solutions in a certain rotating speed ratio region, and in speed-up and speed-down curves, the positions of the "jump" are different;

(3) Decreasing the eccentricity of disks, decreasing eccentricity angle between the two disks, and increasing the damping ratio can shrink the "unstable" region of the response curve; and with the change of the mass ratio, the position of "unstable region" also changes. By adjusting the parameters of the simplified model, the rod-fastened rotor can be effectively prevented from operating in an unstable frequency range. So that, when designs a rotor, the eccentricities of disks and eccentricity angles between the disks should be made as tiny as possible. By doing so, the rotor can operate in a more stable working condition. However, other parameters of the rotor(ie, mass ratio and damping ratio) are reletively harder to change due to other requirements the rotor have to meet. 


\section{References}

[1] Yuan, J., El-Haddad, F., Salles, L. and Wong, C. (2019). Numerical assessment of reduced order modeling techniques for dynamic analysis of jointed structures with contact nonlinearities. Journal Of Engineering For Gas Turbines And Power, 141(3). doi: 10.1115/1.4041147.

[2] Yuan, Q., Gao, J. and Li, P. (2014). Nonlinear Dynamics of the Rod-Fastened Jeffcott Rotor. Journal of Vibration and AcousticsTransactions of the ASME, 136(2). pp. 325-325 doi: 10.1115/1.4026241.

[3] Qin, Z. Y., Han, Q. K., and Chu, F. L. (2014). Analytical model of bolted disk- drum joints and its application to dynamic analysis of jointed rotor. Proceedings of The Institution of Mechanical Engineers Part C-Journal of Mechanical Engineering Science, 228(4), pp. 646-663. doi: 10.1177/0954406213489084.

[4] Liu, Z. Q., Cao, S. Q. and Guo, H. L. (2016). Vibration characteristics of rotor systems with bolt joints. Journal of Vibration and Shock, 35(22), pp. 10-16 and 37. doi: 10.13465/j.cnki.jvs.2016.22.002.

[5] Cheng, L., Li S. and Qian Z. (2011). Bistable vibration characteristics of disk-rod-fastening rotor with squeeze film dumper. Journal of Aerospace Power, 26(7) . pp. 1563-1568. doi: 10.13224/j.cnki.jasp.2013.09.018.

[6] Hei, D, Lu, Y, Zhang, Y, Lu, Z, Gupta, P \& Müller. (2014). Nonlinear dynamic behaviors of a rod fastening rotor supported by fixed-tilting pad journal bearings. Chaos, Solitons and Fractals, 69, pp. 129-150. doi: 10.1016/j.chaos.2014.09.013.

[7] Mat Isa, A. A., Penny, J. E. T. and Garvey, S. D. (2000). Dynamics of bolted and laminated rotors, \#183. Proceedings of the International Modal Analysis Conference - IMAC, 1, pp. 867-872.

[8] Cveticanin, L. (1995). Resonant vibrations of nonlinear rotors. Mechanism and Machine Theory, 30(4), pp. 581-588. doi: 10.1016/0094-114X(94)00059-T.

[9] Wu, J. D., Hou, X. L. and Liu, C. C. (2006). Theoretical analysis of characteristics on main resonance of a rotating system with nonlinear stiffness. China Mechanical Engineering, 17(5), pp. 539-541. doi: 10.3321/j.issn:1004-132X.2006.05.026.

[10] Zhao, M., Ren, P. Z. and Li, J. Q. (2004). Double static analysis of muti-rotor system with squeeze film damper. Journal af Propulsion Technology, 25(1), pp. 66-70. . doi: 10.3321/j.issn:1001-4055.2004.01.018.

[11] Brake, M. R. W., Groß, J., Lacayo, R. M., Salles, L., Schwingshackl, C. W., Reuß, P., and Armand, J.(2018). Reduced Order Modeling of Nonlinear Structures With Frictional Interfaces. In: The Mechanics of Jointed Structures: Recent Research and Open Challenges for Developing Predictive Models for Structural Dynamics, ed. Brake, M.R.W. Cham: Springer International Publishing.

[12] Qin, Z., Han, Q. and Chu, F. (2016). Bolt loosening at rotating joint interface and its influence on rotor dynamics. Engineering Failure Analysis, 59, pp. 456-466. doi: 10.1016/j.engfailanal.2015.11.002.

[13] Krack, M., Salles, L. and Thouverez, F. (2017). Vibration Prediction of Bladed Disks Coupled by Friction Joints. Archives Of Computational Methods In Engineering, 24(3), pp. 589-636. doi: 10.1007/s11831-016-9183-2.

[14] Li, N. C. and Mei, L. Q. (2011). Numerical Analysis. Beijing: Science Press.
[15] Yu, P. C., Ma, Y. H. and Zhang, D. Y. (2016). Dynamic model and vibration characteristic analysis on complex rotor system with local nonlinear stiffness. Journal of Propulsion Technology, 37(12), pp. 2343-2351. doi: 10.13675/j.cnki.tjjs.2016.12.018.

[16] Liu, Y. Z. and Cheng, L. Q. (2001). Nonlinear Vibration. Beijing: Higher Education Press.

\section{NOMENCLATURE}

\begin{tabular}{|c|c|c|}
\hline$l_{1}$ & $=$ & length of segment of disk \\
\hline$l_{2}$ & $=$ & length of shaft \\
\hline$l_{3}$ & $=$ & length of hub \\
\hline$l_{4}$ & $=$ & length of disk \\
\hline$l$ & $=$ & length of model \\
\hline$K_{s}$ & $=$ & bending stiffness of shaft \\
\hline$K_{h}$ & $=$ & bending stiffness of hub \\
\hline$K_{d s}$ & $=$ & bending stiffness of disk \\
\hline$K_{r}$ & $=$ & bending stiffness of rod \\
\hline$K_{c}$ & $=$ & bending stiffness of segment of disk \\
\hline$K_{f i j}$ & $=$ & $\begin{array}{l}\text { moment load applied in the } j \text { direction } \\
\text { when the rotor produces a unit rotation } \\
\text { angle in the } i \text { direction }\end{array}$ \\
\hline$\delta_{i j}$ & $=$ & Kroneck function \\
\hline$K_{c l}$ & $=$ & flexural stiffness of segment of disk \\
\hline$K_{h l}$ & $=$ & flexural stiffness of hub \\
\hline$K_{d s l}$ & $=$ & flexural stiffness of disk \\
\hline$K_{r l}$ & $=$ & $\begin{array}{l}\text { flexural stiffness of rod and contact } \\
\text { surface }\end{array}$ \\
\hline$\varphi$ & $=$ & relative angle of the disks \\
\hline M & $=$ & moment load \\
\hline$\left|\mathrm{K}_{\mathrm{rl}}\right|$ & $=$ & absolute value of $K_{r l}$ \\
\hline$X$ & $=$ & lateral distance between disks \\
\hline$N$ & $=$ & number of tie rods \\
\hline$d_{r}$ & $=$ & diameter of tie rods \\
\hline$E$ & $=$ & Young's modulus of elasticity \\
\hline$\rho$ & $=$ & material density \\
\hline$m$ & $=$ & rotor mass \\
\hline$\zeta$ & $=$ & damping ratio \\
\hline$r_{p}$ & $=$ & pitch radius \\
\hline$F_{p r e}$ & $=$ & total pretightening force \\
\hline${ }_{1}, m_{2}$ & $=$ & the mass of disks \\
\hline$e$ & $=$ & $\begin{array}{l}\text { eccentricity of disks of the simplified } \\
\text { model }\end{array}$ \\
\hline$\theta$ & $=$ & eccentricity angle of the simplified model \\
\hline$F(x)$ & $=$ & $\begin{array}{l}\text { restoring force function of the simplified } \\
\text { model }\end{array}$ \\
\hline$k_{1}, k_{2}$ & $=$ & $\begin{array}{l}\text { flexural stiffness of disks of the simplified } \\
\text { model }\end{array}$ \\
\hline$c_{1}, c_{2}$ & $=$ & damping of shaft of the simplified model \\
\hline$c_{3}$ & $=$ & $\begin{array}{l}\text { damping of segment of disk of the } \\
\text { simplified model }\end{array}$ \\
\hline$q_{1}, q_{2}$ & $=$ & $\begin{array}{l}\text { displacement vector of disks of the } \\
\text { simplified model }\end{array}$ \\
\hline$\Omega$ & $=$ & rotating speed \\
\hline$k_{3}$ & $=$ & $\begin{array}{l}\text { linear stiffness of segment of disk of the } \\
\text { simplified model }\end{array}$ \\
\hline$k_{4}$ & $=$ & $\begin{array}{l}\text { nonlinear stiffness of segment of disk of } \\
\text { the simplified model }\end{array}$ \\
\hline$\tau$ & $=$ & $\Omega t$ \\
\hline
\end{tabular}




$$
\begin{aligned}
r & =\text { Vibration amplitude ratio } \\
r^{\prime} & =\text { first derivative of } r \text { to } \tau \\
r^{\prime \prime} & =\text { second derivative of } r \text { to } \tau \\
\alpha & =\text { mass ratio }\left(=m_{2} / m_{1}\right) \text { of the simplified } \\
& \text { model } \\
\omega_{1} & =\begin{array}{l}
\text { natural frequency of the disk } 1 \text { of the } \\
\text { simplified model }
\end{array} \\
\zeta_{1}, \zeta_{2}, \zeta_{3} & =\text { damping ratio of the simplified model } \\
\lambda & =\text { rotating speed ratio of the simplified } \\
& \text { model } \\
\xi & =\text { stiffness ratio }\left(=e^{2} \beta_{4}\right) \text { of the simplified } \\
& \text { model } \\
r_{1}, r_{2} & =\text { Vibration amplitude ratio of disks of the } \\
& \text { simplified model } \\
r_{3} & =\text { Vibration amplitude difference of disks }(= \\
\hat{r}_{n} & =\text { amplitude of } n \mathrm{x} \text { vibration of } r_{2} \text { or } r_{3} \\
\tilde{r}^{(k)} & =\text { the value of the vector in step } \mathrm{k} \\
f\left(\tilde{r}^{(k)}\right) & =\text { residual of the algebraic equation in step } \mathrm{k} \\
A_{k} & =\text { Jacobian matrix of the algebraic equations } \\
& \text { in step } \mathrm{k} \\
e p s & =\text { residual limit } \\
\Omega_{0}, \Omega_{\text {end }} & =\text { start and end rotating speed } \\
\operatorname{Norm}(\cdot) & =\text { 2-norm }
\end{aligned}
$$

\section{ACKNOWLEDGMENTS}

This work is supported by National Nature Science Foundation of China (No.11372234) 